\title{
CONSTRUCTIVE STABILIZATION OF QUADRATIC-INPUT NONLINEAR SYSTEMS WITH BOUNDED CONTROLS
}

\author{
JIANGHUA ZHONG, DAIZHAN CHENG, and XIAOMING HU \\ ABSTRACT. In this paper, the stabilization of quadratic-input nonlin- \\ ear systems with bounded controls is considered. According to the \\ type of quadratic-input forms, two cases, namely, positive definite \\ and positive semi-definite, are considered. For the case of positive \\ definiteness, a universal formula for bounded stabilizers is given via \\ a known Lyapunov control function. For the case of positive semi- \\ definiteness, a constructive parametrization of bounded stabilizers is \\ proposed under the assumption that there exists a known Lyapunov \\ control function with respect to a smaller control set than the admis- \\ sible control set.
}

\section{INTRODUCTION}

In this paper, we consider the following quadratic-input nonlinear system:

$$
\dot{x}=f(x)+\sum_{i=1}^{m} g_{i}(x) u_{i}+\sum_{i_{1}=1}^{m} \sum_{i_{2}=1}^{m} h_{i_{1} i_{2}}(x) u_{i_{1}} u_{i_{2}},
$$

where $x \in \mathbb{R}^{n}$ is the state,

$$
u \in B(L)=\left\{u \in \mathbb{R}^{m} \mid\|u\|^{2}=u_{1}^{2}+u_{2}^{2}+\cdots+u_{m}^{2}<L\right\}
$$

is the control input, and $f, g$, and $h_{i j}$ are smooth vector fields with $f(0)=0$.

Since the concept of Lyapunov control function was first introduced by Arstein [1], many results have been obtained for the stabilization of nonlinear control systems using the Lyapunov control function approach, e.g., $[2,5-7,9-12]$. Among them, Moulay and Perruquetti gave a formula for the continuous stabilizers for system (1.1) in the single-input case via a known Lyapunov control function [7]. Lin constructed a bounded smooth

2000 Mathematics Subject Classification. 93D15.

Key words and phrases. Quadratic-input nonlinear system, global stabilization, bounded control, Lyapunov control function.

This work is supported partly by NNSF of China under Grants 60221301 and 60334040, partly by Sida-VR Swedish Research Links Grant 348-2002-6936. 
stabilizer for system (1.1) with unbounded controls under one of the assumptions that the unforced system is stable (the asymptotic stability is not necessary; [3, 4]). The authors' recent paper [12] proposed a constructive parametrization of continuous stabilizers for system (1.1) with unbounded controls using Lyapunov control function approach. Suárez et al. constructed a one-parameter family of bounded continuous stabilizers for affine nonlinear control systems with controls taking values in compact convex sets via a known Lyapunov control function [10]. Under the assumption that an appropriate Lyapunov control function is known, Lin and Sontag gave a universal formula for the bounded continuous stabilizers for affine nonlinear control systems with controls taking values in the Euclidean unit ball [5], based on Sontag's formula for continuous stabilizers [9]. Malisoff and Sontag extended Lin and Sontag's result to the Minkowski unit ball [6]. Later, Suárez et al. generalized Malisoff and Sontag's result to a large class of sets of control values [11]. Motivated by these works, this paper considers the stabilization of quadratic-input nonlinear systems with controls taking values from a bounded Euclidean ball. According to the type of quadratic-input forms, two cases, namely, positive definite and positive semi-definite, are considered. For the first case, a universal formula for bounded continuous stabilizers is obtained via the known Lyapunov control function. For the second case, a constructive parametrization of bounded continuous stabilizers is proposed under the assumption that there exists a known Lyapunov control function with respect to a smaller control set than the admissible control set.

The paper is organized as follows. Section 2 presents some preliminaries. Section 3 gives a universal formula for bounded continuous stabilizers for the case of positive definiteness. Section 4 investigates the feasible set of bounded continuous stabilizing controls for the case of positive semidefiniteness. Section 5 provides a detailed bounded control design procedure for the case of positive semi-definiteness. Section 6 gives some illustrative examples. Section 7 is the conclusion.

\section{Preliminaries}

In this section, we recall some basic definitions and facts concerning Lyapunov control functions. We also give preliminary notation and results for system (1.1).

Consider a nonaffine nonlinear control system

$$
\dot{x}=f(x, u),
$$

where $x \in \mathbb{R}^{n}$ is the state, $u \in U \subset \mathbb{R}^{m}$ is the control input, and $f$ is the smooth vector field such that $f(0,0)=0$.

Definition 2.1 (see $[1,7]$ ). A smooth, proper, and positive definite function $V$ is a control Lyapunov function (LCF) for system (2.1) if for 
any $x \in \mathbb{R}^{n} \backslash\{0\}$

$$
\inf _{u \in U}\left\{\frac{\partial V}{\partial x} f(x, u)\right\}<0 .
$$

Definition 2.2 (see $[1,7]$ ). A LCF $V$ for system $(2.1)$ is said to satisfy the small control property if for each $\epsilon>0$, there exists $\delta>0$ such that if $x \neq 0$ satisfies $\|x\|<\delta$, then there exists some $u$ such that $\|u\|<\epsilon$ and

$$
\frac{\partial V}{\partial x} f(x, u)<0 .
$$

From the above definition, one can see that the small control property assures the existence of a stabilizing control $u$ which is continuous at the origin and such that $u(0)=0$.

However, the construction of a bounded continuous stabilizer is, in general, highly nontrivial, except for the affine control case.

We consider an affine nonlinear control system

$$
\dot{x}=f(x)+g(x) u,
$$

where $x \in \mathbb{R}^{n}$ is the state, $u \in B(r)$ is the control input, and $f$ and $g=$ $\left(g_{1}, \ldots, g_{m}\right)$ are smooth vector fields, where $f(0)=0$.

For system (2.4), inequality (2.2) is reduced to

$$
\inf _{u \in B(r)}\left\{\frac{\partial V}{\partial x}\left[f(x)+\sum_{i=1}^{m} g_{i}(x) u_{i}\right]\right\}<0 .
$$

We denote

$$
a(x):=\frac{\partial V}{\partial x} f(x), \quad b_{i}(x):=\frac{\partial V}{\partial x} g_{i}(x), \quad i=1, \ldots, m .
$$

We set

$$
b(x):=\left[b_{1}(x), b_{2}(x), \ldots, b_{m}(x)\right]^{T} .
$$

Then it is easy to see that inequality (2.5) is equivalent to the following expression:

$$
b(x)=0 \Longrightarrow a(x)<0 \forall x \neq 0 .
$$

We denote

$$
k(x):= \begin{cases}-\sqrt{r} \frac{a(x)+\sqrt{a^{2}(x)+\|\tilde{b}(x)\|^{4}}}{\|\tilde{b}(x)\|^{2}\left(1+\sqrt{1+\|\tilde{b}(x)\|^{2}}\right)} \tilde{b}(x) & \text { if } \tilde{b}(x) \neq 0, \\ 0, & \text { if } \tilde{b}(x)=0,\end{cases}
$$

where $\tilde{b}(x)=\sqrt{r} b(x)$.

The following result is from [5] with a slight modification. 
Proposition 2.3. Assume that $V$ is a Lyapunov control function of system (2.4). Then $k(x)$ is smooth everywhere except for the origin, takes values in $B(r)$, and is continuous at the origin if the LCF V satisfies the small control property. Moreover, the control law $u=k(x)$ globally asymptotically stabilizes system (2.4).

Consider system (1.1). Denote by $h_{i_{1} i_{2}}^{i}(x)$ the $i$ th component of $h_{i_{1} i_{2}}(x)$, and for each $i \in\{1, \ldots, n\}$, let

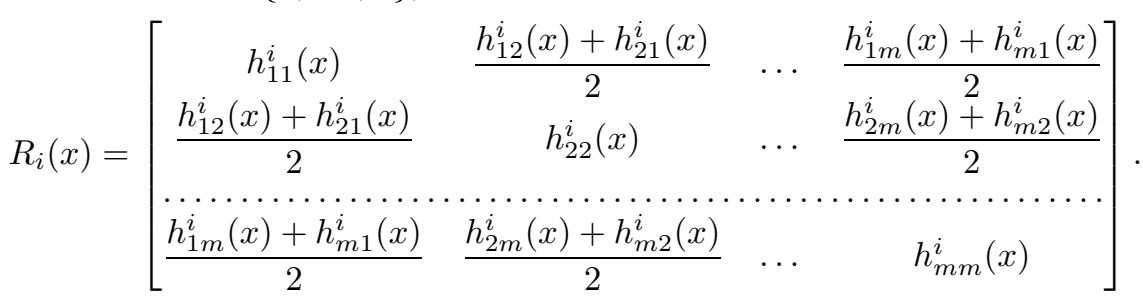

Then

$$
\sum_{i_{1}=1}^{m} \sum_{i_{2}=1}^{m} h_{i_{1} i_{2}}(x) u_{i_{1}} u_{i_{2}}=\left[u^{T} R_{1}(x) u, u^{T} R_{2}(x) u, \ldots, u^{T} R_{n}(x) u\right]^{T} .
$$

Assume that $V$ is a smooth, proper, and positive definite function. Then the time derivative of $V$ along the trajectories of system (1.1) is

$$
\begin{aligned}
\left.\dot{V}\right|_{(1.1)} & =\frac{\partial V}{\partial x} f(x)+\frac{\partial V}{\partial x} g(x) u+\frac{\partial V}{\partial x}\left[u^{T} R_{1}(x) u, u^{T} R_{2}(x) u, \ldots, u^{T} R_{n}(x) u\right]^{T} \\
& =\frac{\partial V}{\partial x} f(x)+\frac{\partial V}{\partial x} g(x) u+\sum_{i=1}^{n}\left(\frac{\partial V}{\partial x_{i}} u^{T} R_{i}(x) u\right) \\
& =\frac{\partial V}{\partial x} f(x)+\frac{\partial V}{\partial x} g(x) u+u^{T}\left(\sum_{i=1}^{n} \frac{\partial V}{\partial x_{i}} R_{i}(x)\right) u .
\end{aligned}
$$

We denote

$$
a(x):=\frac{\partial V}{\partial x} f(x), \quad b(x):=\left(\frac{\partial V}{\partial x} g(x)\right)^{T}, \quad R(x):=\sum_{i=1}^{n} \frac{\partial V}{\partial x_{i}} R_{i}(x) .
$$

Then $R(x)$ is symmetric and

$$
\left.\dot{V}\right|_{(1.1)}=a(x)+b^{T}(x) u+u^{T} R(x) u .
$$

We denote

$$
F(x, u)=a(x)+b^{T}(x) u+u^{T} R(x) u .
$$

Under the assumption that $V$ is a LCF for system (1.1), it has been pointed out in [7] that one of sufficient conditions of the existence of a continuous stabilizer is that $F(x, u)$ is convex with respect to $u$ for all $x \in$ $\mathbb{R}^{n} \backslash\{0\}$ (i.e., $R(x)$ is positive semi-definite except for the origin [12]). In the rest of this paper, we first discuss the case where $R(x)$ is positive definite except for the origin, and then the case where $R(x)$ is positive semi-definite. 


\section{UNIVERSAL FORMULA FOR BOUNDED STABILIZATION}

In this section, we give a universal formula for the bounded continuous stabilizers of system (1.1) via a known Lyapunov control function for the case $R(x)>0$ for all $x \neq 0$, i.e., the case where $R(x)$ is positive definite everywhere except for the origin.

Lemma 3.1 (see [2]). Assume that $A$ is a symmetric, positive definite matrix. Then the set of solutions of the quadratic inequality

$$
\xi^{T} A \xi+d^{T} \xi+c<0,
$$

where $\xi \in \mathbb{R}^{m}$, is nonempty if and only if

$$
\frac{1}{4} d^{T} A^{-1} d-c>0
$$

and the set of solutions is given by

$$
\xi=-\frac{1}{2} A^{-1} d+A^{-\frac{1}{2}} \nu \sqrt{\frac{1}{4} d^{T} A^{-1} d-c},
$$

where $\nu$ satisfies $\|\nu\|<1$.

Using this lemma, we can obtain the following result.

Theorem 3.2. Assume that $V$ is a LCF for system (1.1) and that $R(x)>0$ for all $x \neq 0$. Then a stabilizer $u_{0}=u_{0}(x)$ of system (1.1) such that $u_{0}(0)=0$ can be expressed as follows:

$$
u_{0}(x)=-\frac{1}{2} \frac{\sqrt{L}\left\|R^{1 / 2}(x)\right\|}{\sqrt{L}\left\|R^{1 / 2}(x)\right\|+\sqrt{\frac{1}{4} b^{T}(x) R^{-1}(x) b(x)-a(x)}} R^{-1}(x) b(x)
$$

for all $x \neq 0$, and it takes values in $B(L)$ and is continuous in $\mathbb{R}^{n} \backslash\{0\}$. Moreover, if the LCF $V$ for system (1.1) satisfies the small control property, then $u_{0}(x)$ is continuous in $\mathbb{R}^{n}$.

Proof. Since $V$ is a LCF for system (1.1), there exists $u_{0} \in B(L)$ such that

$$
a(x)+b^{T}(x) u_{0}+u_{0}^{T} R(x) u_{0}<0 \quad \forall x \neq 0,
$$

i.e.,

$$
\left\|R^{1 / 2}(x) u_{0}+\frac{1}{2} R^{-\frac{1}{2}}(x) b(x)\right\|<\sqrt{\frac{1}{4} b^{T}(x) R^{-1}(x) b(x)-a(x)} \quad \forall x \neq 0 .
$$

Note that

$$
\left\|R^{1 / 2}(x) u_{0}+\frac{1}{2} R^{-\frac{1}{2}}(x) b(x)\right\| \geq \frac{1}{2}\left\|R^{-\frac{1}{2}}(x) b(x)\right\|-\left\|R^{1 / 2}(x) u_{0}\right\|
$$

and

$$
\left\|R^{1 / 2}(x) u_{0}\right\| \leq\left\|R^{1 / 2}(x)\right\|\left\|u_{0}\right\|<\sqrt{L}\left\|R^{1 / 2}(x)\right\| .
$$


Then

$$
\frac{1}{2}\left\|R^{-\frac{1}{2}}(x) b(x)\right\|<\sqrt{L}\left\|R^{1 / 2}(x)\right\|+\sqrt{\frac{1}{4} b^{T}(x) R^{-1}(x) b(x)-a(x)}
$$

for all $x \neq 0$. In (3.1), we set

$$
\nu=\nu(x)=\frac{\frac{1}{2} R^{-\frac{1}{2}}(x) b(x)}{\sqrt{L}\left\|R^{1 / 2}(x)\right\|+\sqrt{\frac{1}{4} b^{T}(x) R^{-1}(x) b(x)-a(x)}}
$$

for all $x \neq 0$. In terms of (3.4), $\nu(x)$ in (3.5) satisfies $\|\nu(x)\|<1$ for all $x \neq 0$. By Lemma 3.1, solving inequality (3.3) yields

$$
u_{0}=u_{0}(x)=-\frac{1}{2} \frac{\sqrt{L}\left\|R^{1 / 2}(x)\right\|}{\sqrt{L}\left\|R^{1 / 2}(x)\right\|+\sqrt{\frac{1}{4} b^{T}(x) R^{-1}(x) b(x)-a(x)}} R^{-1}(x) b(x)
$$

for all $x \neq 0$. For any $x \in \mathbb{R}^{n} \backslash\{0\}$, let $v=R^{1 / 2}(x) u_{0}$. Then

$$
v=-\frac{1}{2} \frac{\sqrt{L}\left\|R^{1 / 2}(x)\right\|}{\sqrt{L}\left\|R^{1 / 2}(x)\right\|+\sqrt{\frac{1}{4} b^{T}(x) R^{-1}(x) b(x)-a(x)}} R^{-\frac{1}{2}}(x) b(x)
$$

for all $x \neq 0$. From (3.4), it is easy to see that $\|v\|<\sqrt{L}\left\|R^{1 / 2}(x)\right\|$ for all $x \neq 0$. Therefore, for any $x \in \mathbb{R}^{n} \backslash\{0\}$, there exists $\varpi \in \mathbb{R}^{m}$ satisfying $\|\varpi(x)\|<1$ such that $v=\sqrt{L} R^{1 / 2}(x) \varpi$. In turn, $u_{0}=R^{-\frac{1}{2}}(x) v=\sqrt{L} \varpi$ for all $x \neq 0$. Hence, $\left\|u_{0}\right\|<\sqrt{L}$ for all $x \neq 0$. This completes the proof.

\section{FeAsible SET OF BOUNDED CONTROLS}

In this section, we give a feasible set of bounded continuous stabilizers under certain assumptions for the case of positive semi-definiteness. To assure that the obtained stabilizer $u \in B(L)$, we assume the following.

A1. There exists a smooth, proper, and positive definite function $V$ such that

$$
\inf _{u \in B(L / 2)}\left[a(x)+b^{T}(x) u+u^{T} R(x) u\right]<0 \quad \forall x \neq 0 .
$$

It is easy to see that if assumption A1 holds, then $V$ is a LCF for system (1.1). But the inverse may not be true. Thus, assumption A1 is stronger than the assumption that $V$ is a LCF for system (1.1).

Under assumption A1, we define

$$
\xi(x):=\inf _{u \in B(L / 2)}\left[a(x)+b^{T}(x) u+u^{T} R(x) u\right] .
$$

Then $\xi(x) \in[-\infty, 0)$ for all $x \neq 0$, and $\xi(0)=0$ since $a(0)=0, b(0)=0$, and $R(0)=0$. For any $\alpha<0$, we define truncated $\xi$ as follows:

$$
\xi_{\alpha}(x)=\max \{\xi(x), \alpha\} .
$$

It is easy to see that if $\xi(x)$ is continuous, then $\xi_{\alpha}(x)$ is also a continuous function. 
First, we need the following lemma.

Lemma 4.1. $B(L / 2) \subset[B(L / 2) \cap \operatorname{Im}(R(x))]+\left[B(L / 2) \cap \operatorname{Im}^{\perp}(R(x))\right]$.

Proof. For any $u \in B(L / 2) \subset \mathbb{R}^{m}, u=u_{I}+u_{P}$, where $u_{I} \in \operatorname{Im}(R(x))$ and $u_{P} \in \operatorname{Im}^{\perp}(R(x))$. Note that $\|u\|^{2}=\left\|u_{I}\right\|^{2}+\left\|u_{P}\right\|^{2}$ and $\|u\|^{2}<L / 2$. Then $\left\|u_{I}\right\|^{2}<L / 2$ and $\left\|u_{P}\right\|^{2}<L / 2$. The lemma is proved.

Now, for each fixed $x$, we decompose $u$ as follows:

$$
u(x)=u_{I}(x)+u_{P}(x),
$$

where

$$
u_{I}(x) \in \operatorname{Im}(R(x)), \quad u_{P}(x) \in \operatorname{Im}^{\perp}(R(x)) .
$$

Then

$$
a(x)+b^{T}(x) u+u^{T} R(x) u=a(x)+b^{T}(x) u_{I}+b^{T}(x) u_{P}+u_{I}^{T} R(x) u_{I} .
$$

Under assumption A1, we also define

$$
\eta(x):=\inf _{u_{I} \in B(L / 2) \cap \operatorname{Im}(R(x))}\left[a(x)+b^{T}(x) u_{I}+u_{I}^{T} R u_{I}\right] .
$$

Note that $\eta(x)>-\infty$ is a well-posed function.

We denote

$$
\left.D=\left\{f \in C_{0}\left(\mathbb{R}^{n} \backslash\{0\}\right) \mid 0<f(x)<1\right)\right\},
$$

i.e., $D$ is the set of continuous functions in $\mathbb{R}^{n} \backslash\{0\}$ with values in $(0,1)$. Choosing any $\alpha<0$ and any $\mu(x) \in D$, we have

$$
\begin{aligned}
& \quad \inf _{u \in B(L / 2)}\left[a(x)+b^{T}(x) u+u^{T} R(x) u\right] \\
& \geq \operatorname{lnf}_{u_{I} \in B(L / 2) \cap \operatorname{Im}(R(x))}\left[a(x)+b^{T}(x) u_{I}+u_{I}^{T} R(x) u_{I}-\eta(x)+\mu(x) \xi_{\alpha}(x)\right] \\
& \quad+\inf _{u_{P} \in B(L / 2) \cap \operatorname{Im}^{\perp}(R(x))}\left[\eta(x)-\mu(x) \xi_{\alpha}(x)+b^{T}(x) u_{P}\right] .
\end{aligned}
$$

For the first term of the right hand side of (4.5), we have

$$
\begin{array}{r}
\inf _{u_{I} \in B(L / 2) \cap \operatorname{Im}(R(x))}\left[a(x)+b^{T}(x) u_{I}+u_{I}^{T} R(x) u_{I}-\eta(x)+\mu(x) \xi_{\alpha}(x)\right] \\
=\mu(x) \xi_{\alpha}(x)<0 \quad \forall x \neq 0 .
\end{array}
$$

For the second term, we have

$$
\begin{array}{r}
\inf _{u_{P} \in B(L / 2) \cap \operatorname{Im}^{\perp}(R(x))}\left[\eta(x)-\mu(x) \xi_{\alpha}(x)+b^{T}(x) u_{P}\right] \leq \xi(x)-\mu(x) \xi_{\alpha}(x) \\
\leq(1-\mu(x)) \xi_{a}(x)<0 \quad \forall x \neq 0 .
\end{array}
$$

Next, we can construct bounded continuous controls $u_{I}$ and $u_{P}$ separately. Then $u=u_{I}+u_{P}$ is a feasible bounded control. 
We set

$$
\begin{aligned}
& a_{1}(x)=a(x)-\eta(x)+\mu(x) \xi_{\alpha}(x), \\
& a_{2}(x)=\eta(x)-\mu(x) \xi_{\alpha}(x) .
\end{aligned}
$$

From inequalities (4.6) and (4.7) we obtain the following result.

Lemma 4.2. Assume that assumption A1 holds. Then there exist $u_{I} \in$ $B(L / 2) \cap \operatorname{Im}(R(x))$ and $u_{P} \in B(L / 2) \cap \operatorname{Im}^{\perp}(R(x))$ such that

$$
a_{1}(x)+b^{T}(x) u_{I}+u_{I}^{T} R(x) u_{I}<0 \quad \forall x \neq 0
$$

and

$$
a_{2}(x)+b^{T}(x) u_{P}<0 \quad \forall x \neq 0 .
$$

Remark. This lemma yields that as long as

$$
\inf _{u \in B(L / 2)}\left[a(x)+b^{T}(x) u+u^{T} R(x) u\right]<0 \quad \forall x \neq 0,
$$

we have

$$
\inf _{u_{I} \in B(L / 2) \cap \operatorname{Im}(R(x))}\left[a_{1}(x)+b^{T}(x) u_{I}+u_{I}^{T} R(x) u_{I}\right]<0 \quad \forall x \neq 0
$$

and

$$
\inf _{u_{P} \in B(L / 2) \cap \operatorname{Im}^{\perp}(R(x))}\left[a_{2}(x)+b^{T}(x) u_{P}\right]<0 \quad \forall x \neq 0 .
$$

To construct $u(x)$, we also need the following lemma.

Lemma 4.3. If $u_{I}(x) \in B(L / 2) \cap \operatorname{Im}(R(x))$ is a continuous (except possibly for the origin) control satisfying inequality (4.8) and $u_{P}(x) \in$ $B(L / 2) \cap \operatorname{Im}^{\perp}(R(x))$ is a continuous (except possibly for the origin) control satisfying inequality (4.9), then $u(x)=u_{I}(x)+u_{P}(x)$ is also continuous (except possibly for the origin) and satisfies $\|u\|<\sqrt{L}$ and

$$
a(x)+b^{T}(x) u(x)+u(x)^{T} R(x) u(x)<0 \quad \forall x \neq 0 .
$$

Proof. The continuity and boundedness of $u$ are obvious. Therefore, it suffices to prove inequality (4.13):

$$
\begin{aligned}
a(x)+ & b^{T}(x) u(x)+u(x)^{T} R(x) u(x) \\
= & a(x)+b(x)\left(u_{I}+u_{P}\right)+\left(u_{I}+u_{P}\right)^{T} R\left(u_{I}+u_{P}\right) \\
= & a(x)+b^{T}(x) u_{I}+b^{T}(x) u_{P}+u_{I}^{T} R u_{I} \\
= & {\left[a(x)+b^{T}(x) u_{I}+u_{I}^{T} R u_{I}-\eta(x)+\mu(x) \xi_{\alpha}(x)\right] } \\
& \quad+\left[\eta(x)-\mu(x) \xi_{\alpha}(x)+b^{T}(x) u_{P}\right] \\
= & {\left[a_{1}(x)+b^{T}(x) u_{I}+u_{I}^{T} R u_{I}\right]+\left[a_{2}(x)+b^{T}(x) u_{P}\right]<0 \quad \forall x \neq 0 . }
\end{aligned}
$$

The proof is complete. 
Finally, under assumption A1, we construct three feasible sets as follows:

$$
\begin{aligned}
& \Phi_{\mu}^{\alpha}=\{\left\{u_{I}(x) \in B(L / 2) \cap \operatorname{Im}(R(x)) \mid\right. \\
&\left.a_{1}(x)+b^{T}(x) u_{I}(x)+u_{I}^{T}(x) R(x) u_{I}(x)<0 \forall x \neq 0 ; u_{I}(0)=0\right\}, \\
& \Psi_{\mu}^{\alpha}=\left\{u_{P}(x) \in B(L / 2) \cap \operatorname{Im}^{\perp}(R(x)) \mid\right. \\
&\left.a_{2}(x)+b^{T}(x) u_{P}(x)<0 \forall x \neq 0 ; u_{P}(0)=0\right\}, \\
& \Phi=\{u(x) \in B(L) \mid \\
&\left.a(x)+b^{T}(x) u(x)+u^{T}(x) R(x) u(x)<0 \forall x \neq 0 ; u(0)=0\right\} .
\end{aligned}
$$

Based on the above arguments, after some tedious but straightforward calculations that are omitted here, we obtain the first main result in the following theorem. It provides a complete parametrized expression of the feasible set of $u$, from which the bounded continuous stabilizing controls can be constructed.

Theorem 4.4. Assume the A1 holds. Then the feasible set of bounded stabilizing controls u for system (1.1) is as follows:

$$
\Phi=\bigcup_{\alpha<0} \bigcup_{\mu(x) \in D}\left\{u=u_{I}(x)+u_{P}(x) \mid u_{I}(x) \in \Phi_{\mu}^{\alpha}, u_{P}(x) \in \Psi_{\mu}^{\alpha}\right\} .
$$

Proof. Note that the choices of the parameters $\alpha$ and $\mu(x)$ are independent. According to Lemmas 4.2 and 4.3, $\Phi$ is composed of double inclusions.

\section{Bounded Continuous CONTROL DESign}

In this section, we construct bounded continuous $u_{I}(x)$ from inequality (4.11) and bounded continuous $u_{P}(x)$ from inequality (4.12) in the case of $R(x) \geq 0$, i.e., the case where $R(x)$ is positive semi-definite. We assume the following.

A2. $R(x) \geq 0$.

For the sake of presentation ease, we also tentatively assume the following.

A3.

1. For any $\alpha<0, \xi_{\alpha}(x)$ is continuous in $\mathbb{R}^{n} \backslash\{0\}$;

2. $\eta(x)$ is continuous in $\mathbb{R}^{n} \backslash\{0\}$.

First, we consider inequality (4.11). Under assumption A2, it is similar to the case where $R(x)>0$ for all $x \neq 0$ discussed in Sec. 3. However, due to the restriction on $u_{I}$, certain further investigation is needed. In the sequel, we denote by $A^{+}$the pseudo-inverse of $A$ (see [8]). 
The following is an easily verifiable result.

Lemma 5.1 (see [12]). Let $A$ be a symmetric $(n \times n)$-matrix, $u \in \mathbb{R}^{n}$, and $v=A u$. Then $u=A^{+} v$ if and only if $u \in \operatorname{Im}(A)$.

We need one more assumption in order to construct continuous controls.

A4. $\left(R^{1 / 2}\right)^{+}(x)$ is continuous in $\mathbb{R}^{n} \backslash\{0\}$.

Note that if we want $u_{I}$ and $u_{P}$ to be continuous, then the assumptions in A3 become necessary. First, we should show that assumptions in A3 are reasonable. First, we give the following result.

Lemma 5.2. Assume that $\mathrm{A} 1$ and $\mathrm{A} 2$ hold and that $u_{I} \in \operatorname{Im}(R(x))$. Let $v=R^{1 / 2}(x) u_{I}$. Then

$$
\left\|u_{I}\right\|<\sqrt{\frac{L}{2}} \Longleftrightarrow\|v\|<\sqrt{\frac{L}{2}}\left\|R^{1 / 2}(x)\right\| .
$$

Proof. Necessity.

$$
\|v\| \leq\left\|R^{1 / 2}(x)\right\|\left\|u_{I}\right\|<\sqrt{\frac{L}{2}}\left\|R^{1 / 2}(x)\right\| .
$$

Sufficiency. Note that $\|v\|<\sqrt{L / 2}\left\|R^{1 / 2}(x)\right\|$ if and only if $v=$ $\sqrt{L / 2} R^{1 / 2}(x) \omega$ for some $\omega \in \mathbb{R}^{m}$ satisfying $\|\omega\|<1$. Then along with Lemma 5.1, $u_{I}=\sqrt{L / 2}\left(R^{1 / 2}\right)^{+}(x) R^{1 / 2}(x) \omega$. Since $\left\|\left(R^{1 / 2}\right)^{+}(x) R^{1 / 2}(x)\right\| \leq$ 1 for all $x \in \mathbb{R}^{n}$,

$$
\left\|u_{I}\right\| \leq \sqrt{\frac{L}{2}}\left\|\left(R^{1 / 2}\right)^{+}(x) R^{1 / 2}(x)\right\|\|\omega\|<\sqrt{\frac{L}{2}} .
$$

The lemma is proved.

Now we want to show that assumption A4 is a sufficient condition for Assumption A3.

Lemma 5.3. If assumptions $\mathrm{A} 1, \mathrm{~A} 2$, and $\mathrm{A} 4$ hold, then $\eta(x)$ and $\xi(x)$ are continuous in $\mathbb{R}^{n} \backslash\{0\}$.

Proof. Recall that

$$
\eta(x)=\inf _{u_{I} \in B(L / 2) \cap \operatorname{Im}(R(x))}\left[a(x)+b^{T}(x) u_{I}+u_{I}^{T} R(x) u_{I}\right] .
$$

We set $v=R^{1 / 2}(x) u_{I}$. Then by Lemma $5.1, u_{I}=\left(R^{1 / 2}\right)^{+}(x) v$. Hence

$$
\begin{aligned}
a(x)+b^{T}(x) u_{I}+ & u_{I}^{T} R(x) u_{I} \\
=a(x)+b^{T}(x)\left(R^{1 / 2}\right)^{+}(x) v+v^{T} v & \\
=\left[v+\frac{1}{2}\left(R^{1 / 2}\right)^{+}(x) b(x)\right]^{T} & {\left[v+\frac{1}{2}\left(R^{1 / 2}\right)^{+}(x) b(x)\right] } \\
& +a(x)-\frac{1}{4} b^{T}(x) R^{+}(x) b(x) .
\end{aligned}
$$


On the other hand, by Lemma 5.2,

$$
\|v\|<\sqrt{\frac{L}{2}}\left\|R^{1 / 2}(x)\right\| .
$$

Therefore,

1. if

then

$$
\frac{1}{2}\left\|\left(R^{1 / 2}\right)^{+}(x) b(x)\right\| \leq \sqrt{\frac{L}{2}}\left\|R^{1 / 2}(x)\right\|,
$$

2. if

$$
\eta(x)=a(x)-\frac{1}{4} b^{T}(x) R^{+}(x) b(x)
$$

then

$$
\frac{1}{2}\left\|\left(R^{1 / 2}\right)^{+}(x) b(x)\right\|>\sqrt{\frac{L}{2}}\left\|R^{1 / 2}(x)\right\|,
$$

$$
\begin{aligned}
& \eta(x)=\left.\left[a(x)+b^{T}(x)\left(R^{1 / 2}\right)^{+}(x) v+v^{T} v\right]\right|_{v=-\sqrt{\frac{L}{2}} R^{1 / 2}(x) \frac{\left(R^{1 / 2}\right)+(x) b(x)}{\left\|\left(R^{1 / 2}\right)^{+}(x) b(x)\right\|}} \\
& =a(x)-\sqrt{\frac{L}{2}} \frac{b^{T}(x)\left(R^{1 / 2}\right)^{+}(x) b(x)}{\left\|\left(R^{1 / 2}\right)^{+}(x) b(x)\right\|}+\frac{L}{2} \frac{b^{T}(x)\left(R^{1 / 2}\right)^{+}(x) R^{1 / 2}(x) b(x)}{\left\|\left(R^{1 / 2}\right)^{+}(x) b(x)\right\|^{2}} .
\end{aligned}
$$

Summarizing the above, we have

$$
\eta(x)=\left\{\begin{aligned}
a(x)- & \frac{1}{4} b^{T}(x) R^{+}(x) b(x) \\
& \text { if } \frac{1}{2}\left\|\left(R^{1 / 2}\right)^{+}(x) b(x)\right\| \leq \sqrt{\frac{L}{2}}\left\|R^{1 / 2}(x)\right\|, \\
a(x)- & \sqrt{\frac{L}{2}} \frac{b^{T}(x)\left(R^{1 / 2}\right)^{+}(x) b(x)}{\left\|\left(R^{1 / 2}\right)^{+}(x) b(x)\right\|} \\
+ & \frac{L}{2} \frac{b^{T}(x)\left(R^{1 / 2}\right)^{+}(x) R^{1 / 2}(x) b(x)}{\left\|\left(R^{1 / 2}\right)^{+}(x) b(x)\right\|^{2}} \\
& \text { if } \frac{1}{2}\left\|\left(R^{1 / 2}\right)^{+}(x) b(x)\right\|>\sqrt{\frac{L}{2}}\left\|R^{1 / 2}(x)\right\| .
\end{aligned}\right.
$$

Hence it is easy to see that if $\left(R^{1 / 2}\right)^{+}(x)$ is continuous in $\mathbb{R}^{n} \backslash\{0\}$, then so is $\eta(x)$.

Next, we consider $\xi(x)$. Note that

$$
\xi(x)=\inf _{u \in B(L / 2)}\left[a(x)+b^{T}(x) u+u^{T} R(x) u\right] .
$$

According to the proof of Lemma 4.1, for any $u \in B(L / 2), u=u_{I}+u_{P}$, where $u_{I} \in B(L / 2) \cap \operatorname{Im}(R(x)), u_{P} \in B(L / 2) \cap \operatorname{Im}^{\perp}(R(x))$. Therefore,

$$
\begin{aligned}
F(x, u)=a(x)+b^{T}(x) u+ & u^{T} R(x) u \\
& =a(x)+b^{T}(x) u_{I}+u_{I}^{T} R(x) u_{I}+b^{T}(x) u_{P} .
\end{aligned}
$$


Represent $b(x)$ as

$$
b(x)=b_{I}(x)+b_{P}(x)
$$

where $b_{I} \in \operatorname{Im}(R(x))$ and $b_{P} \in \operatorname{Im}^{\perp}(R(x))$. Let $v=R^{1 / 2}(x) u_{I}$. Then by Lemma 5.1, $u_{I}=\left(R^{1 / 2}\right)^{+}(x) v$. We set $u_{I}=(\|u\| \cos \theta) e_{I}$ and $u_{P}=$ $(\|u\| \sin \theta) e_{P}$, where $\theta$ is the angle between $u$ and $u_{I}$ and $\left\|e_{I}\right\|=\left\|e_{p}\right\|=1$. Hence

$$
\begin{aligned}
F(x, u)=a(x)+ & b^{T}(x) u+u^{T} R(x) u \\
=a(x)+ & b^{T}(x)\left(R^{1 / 2}\right)^{+}(x) v+v^{T} v+\left(b_{I}^{T}(x)+b_{P}^{T}(x)\right) u_{P} \\
= & \left.+v+\frac{1}{2}\left(R^{1 / 2}\right)^{+}(x) b(x)\right]^{T}\left[v+\frac{1}{2}\left(R^{1 / 2}\right)^{+}(x) b(x)\right] \\
& \quad+a(x)-\frac{1}{4} b^{T}(x) R^{+}(x) b(x)+b_{P}^{T}(x) u_{P} \\
=\| & (\|u\| \cos \theta) R^{1 / 2}(x) e_{I}+\frac{1}{2}\left(R^{1 / 2}\right)^{+}(x) b(x) \|^{2} \\
& \quad+(\|u\| \sin \theta) b_{P}^{T}(x) e_{P}+a(x)-\frac{1}{4} b^{T}(x) R^{+}(x) b(x) .
\end{aligned}
$$

In terms of $(5.4)$, to make $F(x, u)$ minimum with respect to $u$, we should have $\|u\|=\sqrt{L / 2}$. Note that $0 \leq \theta \leq \pi / 2$. Then we have the following results.

Case 1. If $\theta=0$ (i.e., $u_{P}=0$ ) or $b_{P}(x)=0$, then $\xi(x)=\eta(x)$.

Case 2. If $\theta=\pi / 2$ (i.e., $u_{I}=0$ ), then $\xi(x)=a(x)-\sqrt{L / 2}\left\|b_{P}(x)\right\|$.

Case 3. If $R^{+}(x) b(x)=0$, then $R(x) b(x)=R^{2}(x) R^{+}(x) b(x)=0$ and $b^{T}(x) R(x) b(x)=0$. Therefore, $b(x) \in \operatorname{Im}^{\perp}(R(x))$. In turn, $b^{T}(x) u_{I}=0$. Note that $u_{I} R(x) u_{I} \geq 0$. Then $\xi(x)=a(x)-\sqrt{L / 2}\left\|b_{P}(x)\right\|$.

Case 4. If $R^{+}(x) b(x) \neq 0,0<\theta<\pi / 2$, and $b_{P}(x) \neq 0$, then to make $F(x, u)$ minimum with respect to $u$, we should have

$$
e_{I}=-R^{+}(x) b(x) /\left\|R^{+}(x) b(x)\right\|, \quad e_{P}=-b_{P}(x) /\left\|b_{P}(x)\right\| \text {. }
$$

Hence the minimum of $F(x, u)$ with respect to $u$ is reduced to the minimum of $\bar{F}(x, \theta)$ with respect to $\theta$, where $0<\theta<\pi / 2$ and

$$
\begin{aligned}
\bar{F}(x, \theta)=-\sqrt{\frac{L}{2}} & \frac{b^{T}(x) R^{+}(x) b(x)}{\left\|R^{+}(x) b(x)\right\|} \cos \theta \\
& +\frac{L}{2} \frac{b^{T}(x) R^{+}(x) b(x)}{\left\|R^{+}(x) b(x)\right\|^{2}} \cos ^{2} \theta-\sqrt{\frac{L}{2}}\left\|b_{P}(x)\right\| \sin \theta+a(x) .
\end{aligned}
$$


Denote

$$
\begin{gathered}
\tau=\tau(x)=\sqrt{\frac{L}{2}} \frac{b^{T}(x) R^{+}(x) b(x)}{\left\|R^{+}(x) b(x)\right\|}, \\
\beta=\beta(x)=\frac{L}{2} \frac{b^{T}(x) R^{+}(x) b(x)}{\left\|R^{+}(x) b(x)\right\|^{2}}, \\
\gamma=\gamma(x)=\sqrt{\frac{L}{2}}\left\|b_{P}(x)\right\|,
\end{gathered}
$$

and

$$
z=\cos \theta, \quad 0<\theta<\pi / 2
$$

Then

$$
\tilde{F}(x, z):=\bar{F}(x, \theta)=-\tau(x) z+\beta(x) z^{2}-\gamma(x) \sqrt{1-z^{2}}+a(x),
$$

where $\tau, \beta, \gamma>0$ and $0<z<1$. Hence the minimum of $\bar{F}(x, \theta)$ with respect to $\theta$ is reduced to that of $\tilde{F}(x, z)$ with respect to $z$. Obviously,

$$
\begin{gathered}
\frac{\partial \tilde{F}}{\partial z}=-\tau+2 \beta z+\frac{\gamma z}{\sqrt{1-z^{2}}}, \\
\frac{\partial^{2} \tilde{F}}{\partial z^{2}}=2 \beta+\frac{\gamma}{\left(1-z^{2}\right) \sqrt{1-z^{2}}}>0
\end{gathered}
$$

for all $z \in(0,1)$. Moreover, note that

$$
\lim _{z \rightarrow 0^{+}} \frac{\partial \tilde{F}}{\partial z}=-\tau<0, \quad \lim _{z \rightarrow 1^{-}} \frac{\partial \tilde{F}}{\partial z}=+\infty .
$$

Therefore, we conclude that there exists a unique $z_{0} \in(0,1)$ such that

$$
\frac{\partial \tilde{F}}{\partial z}=0
$$

and that the minimum of $\tilde{F}(x, z)$ with respect to $z$ is $\tilde{F}\left(x, z_{0}\right)$. Solving

$$
\frac{\partial \tilde{F}}{\partial z}=0
$$

with respect to $z$ is reduced to solving the equation

$$
4 \beta z^{4}-4 \tau \beta z^{3}+\left(\tau^{2}-4 \beta^{2}+\gamma^{2}\right) z^{2}+4 \tau \beta z-\tau^{2}=0,
$$

which can be solved in the existing formulas. Therefore, $\xi(x)=\tilde{F}\left(x, z_{0}\right)$, where $z_{0}$ is the real solution of Eq. (5.5) taking values in $(0,1)$.

Summarizing the above, we have

$$
\xi(x)=\min \left\{\eta(x), a(x)-\sqrt{\frac{L}{2}}\left\|b_{P}(x)\right\|, \tilde{F}\left(x, z_{0}\right)\right\} .
$$

Hence if $\left(R^{1 / 2}\right)^{+}(x)$ is continuous in $\mathbb{R}^{n} \backslash\{0\}$, then so is $\eta(x)$. 
We set $v=R^{1 / 2}(x) u_{I}$. Note that $\operatorname{Im}\left(R^{1 / 2}(x)\right)=\operatorname{Im}(R(x))$ and, therefore, according to Lemma 5.1,

$$
u_{I}=\left(R^{1 / 2}\right)^{+}(x) v \in \operatorname{Im}(R(x)) .
$$

Moreover, for each $u_{I} \in \operatorname{Im}(R(x))$, we can find $v$ such that

$$
u_{I}=\left(R^{1 / 2}\right)^{+}(x) v .
$$

We denote

$$
\mathcal{S}=\left\{v \in \mathbb{R}^{m} \mid\|v\|^{2}=v_{1}^{2}+v_{2}^{2}+\cdots+v_{m}^{2}<\frac{L}{2}\left\|R^{1 / 2}(x)\right\|^{2}\right\} ;
$$

then, along with Lemma 5.2, we can rewrite inequality (4.11) as follows:

$$
\inf _{v \in \mathcal{S}}\left[a_{1}(x)+\tilde{b}^{T}(x) v+v^{T} v\right]<0 \quad \forall x \neq 0,
$$

where $\tilde{b}(x)=\left(R^{1 / 2}\right)^{+}(x) b(x)$. Thus, the solutions of inequality (5.6) and the solutions of inequality (4.11) are in a one-to-one correspondence $v \leftrightarrow u_{I}=$ $\left(R^{1 / 2}\right)^{+}(x) v$. Now obtaining the parametrized formula of $v(x)$ satisfying inequality (5.6) is similar to the case of $R(x)>0$ for all $x \neq 0$, which was discussed in the previous section. Therefore, we have the following theorem.

Theorem 5.4. Assume that A1, A2, and A4 hold. Then a feasible control $u_{I}$ with $u_{I}(0)=0$ is given by

$$
u_{I}(x)=-\frac{1}{2} \frac{\sqrt{\frac{L}{2}}\left\|R^{1 / 2}(x)\right\|}{\sqrt{\frac{L}{2}}\left\|R^{1 / 2}(x)\right\|+\sqrt{\frac{1}{4} b^{T}(x) R^{+}(x) b(x)-a_{1}(x)}} R^{+}(x) b(x)
$$

for all $x \neq 0$, which takes values in $B(L / 2)$ and is continuous in $\mathbb{R}^{n} \backslash\{0\}$. Moreover, if the LCF $V$ for system (1.1) satisfies the small control property, then $u_{I}(x)$ is continuous in $\mathbb{R}^{n}$.

Proof. In terms of inequality (4.11), there exists $u_{I} \in B(L / 2) \cap \operatorname{Im}(R(x))$ such that

$$
a_{1}(x)+b^{T}(x) u_{I}+u_{I}^{T} R(x) u_{I}<0 \quad \forall x \neq 0,
$$

i.e.,

$$
\left\|R^{1 / 2}(x) u_{I}+\frac{1}{2}\left(R^{1 / 2}\right)^{+}(x) b(x)\right\|<\sqrt{\frac{1}{4} b^{T}(x) R^{+}(x) b(x)-a_{1}(x)}
$$

for all $x \neq 0$. Note that

$$
\left\|R^{1 / 2}(x) u_{I}+\frac{1}{2}\left(R^{1 / 2}\right)^{+}(x) b(x)\right\| \geq \frac{1}{2}\left\|\left(R^{1 / 2}\right)^{+}(x) b(x)\right\|-\left\|R^{1 / 2}(x) u_{I}\right\|
$$

and

$$
\left\|R^{1 / 2}(x) u_{I}\right\| \leq\left\|R^{1 / 2}(x)\right\|\left\|u_{I}\right\|<\sqrt{\frac{L}{2}}\left\|R^{1 / 2}(x)\right\| .
$$


Then

$$
\begin{aligned}
\frac{1}{2} \|\left(R^{1 / 2}\right)^{+} & (x) b(x) \| \\
& <\sqrt{\frac{L}{2}}\left\|R^{1 / 2}(x)\right\|+\sqrt{\frac{1}{4} b^{T}(x) R^{+}(x) b(x)-a_{1}(x)}
\end{aligned}
$$

for all $x \neq 0$. On the other hand, since assumptions A1 and A2 hold, by the previous arguments, inequality (5.6) holds. Hence there exists $v \in \mathcal{S}$ such that

$$
a_{1}(x)+\tilde{b}^{T}(x) v+v^{T} v<0 \quad \forall x \neq 0 .
$$

By Lemma 3.1, solving the above inequality, we have

$$
\begin{aligned}
& v=-\frac{1}{2} \tilde{b}(x)+\nu(x) \sqrt{\frac{1}{4} \tilde{b}^{T}(x) \tilde{b}(x)-a_{1}(x)} \\
&=-\frac{1}{2}\left(R^{1 / 2}\right)^{+}(x) b(x)+\nu(x) \sqrt{\frac{1}{4} b^{T}(x) R^{+}(x) b(x)-a_{1}(x)},
\end{aligned}
$$

$\|\nu(x)\|<1$ for all $x \neq 0$.

To assure that $v \in \mathcal{S}$, we choose

$$
\nu(x)=\frac{\frac{1}{2}\left(R^{1 / 2}\right)^{+}(x) b(x)}{\sqrt{\frac{L}{2}}\left\|R^{1 / 2}(x)\right\|+\sqrt{\frac{1}{4} b^{T}(x) R^{+}(x) b(x)-a_{1}(x)}}
$$

for all $x \neq 0$. From (5.8), we can see that $\nu(x)$ of the form (5.10) satisfies $\|\nu(x)\|<1$, for all $x \neq 0$. Substituting (5.10) into (5.9), we have

$$
v=-\frac{1}{2} \frac{\sqrt{\frac{L}{2}}\left\|R^{1 / 2}(x)\right\|}{\sqrt{\frac{L}{2}}\left\|R^{1 / 2}(x)\right\|+\sqrt{\frac{1}{4} b^{T}(x) R^{+}(x) b(x)-a_{1}(x)}}\left(R^{1 / 2}\right)^{+}(x) b(x)
$$

for all $x \neq 0$. Since (5.8) holds, it is easy to verify that $v$ of the form (5.11) satisfies $\|v\|<\sqrt{L / 2}\left\|R^{1 / 2}(x)\right\|$ for all $x \neq 0$. Then $u_{I}=\left(R^{1 / 2}\right)^{+}(x) v$ yields (5.7). According to Lemma 5.2, $\left\|u_{I}\right\|<\sqrt{L / 2}$ for all $x \neq 0$. This completes the proof.

Next, we deduce $u_{P}$ from inequality (4.12). We will also relax the restriction on $u_{P}$.

Lemma 5.5. Let $A$ be a symmetric $(n \times n)$-matrix. Define

$$
P_{A}:=I_{n}-A^{+} A \text {. }
$$

Then

$$
P_{A}: \mathbb{R}^{n} \rightarrow \mathbb{R}^{n}, \quad x \mapsto P_{A} x,
$$

satisfies the following conditions:

1. $\operatorname{Im}\left(P_{A}\right)=I_{m}^{\perp}(A)$; 
2. $\left.P_{A}\right|_{\operatorname{Im}^{\perp}(A)}=$ identity.

Proof. 1. We show only that $\operatorname{Im}\left(P_{A}\right) \subset \operatorname{Im}^{\perp}(A)$. The "equality" follows from the following proof of the second statement.

Let $x \in \mathbb{R}^{n}$, we have only to show that $\left(P_{A} x\right)^{T} A=0$. Since $A$ is symmetric,

$$
\left(P_{A} x\right)^{T} A=x^{T}\left(I-A A^{+}\right) A=x^{T}\left[A-A A^{+} A\right]=0 .
$$

2. Suppose that $\operatorname{rank}(A)=s$. Then there exists an orthogonal $(n \times n)$ matrix $P$ such that

$$
P A P^{T}=\operatorname{diag}\left(\lambda_{1}, \ldots, \lambda_{s}, 0, \ldots, 0\right),
$$

where $\lambda_{i}, i=1, \ldots, s$, are nonzero eigenvalues of $A$.

For any $i \in\{1, \ldots, s\}$, denote by $\xi_{i}$ the eigenvector of $A$ corresponding to $\lambda_{i}$. Hence for any $y \in \operatorname{Im}(A)$, we have $y \in \operatorname{Span}\left\{\xi_{1}, \ldots, \xi_{s}\right\}$. Note that $P \xi_{i}$, $i=1, \ldots, s$, are eigenvectors of $P A P^{T}$ with respect to $\lambda_{i}$ and, therefore, $P \xi_{i}=k_{i} \delta_{i}$, where $\delta_{i}$ is the $i$ th column of $I_{n}, k_{i} \neq 0, i=1, \ldots, s$. Since $P y \in \operatorname{Span}\left\{P \xi_{1}, \ldots, P \xi_{s}\right\}$,

$$
P y=\left[y_{1}, \ldots, y_{s}, 0, \ldots, 0\right]^{T} .
$$

For any $x \in \operatorname{Im}^{\perp}(A)$, note that

$$
(P x)^{T} P y=x^{T} P^{T} P y=x^{T} y=0 \quad \forall y \in \operatorname{Im}(A),
$$

and, therefore,

$$
P x=\left[0, \ldots, 0, x_{s+1}, \ldots, x_{n}\right]^{T} .
$$

On the other hand, if

$$
P x=\left[0, \ldots, 0, x_{s+1}, \ldots, x_{n}\right]^{T} \quad \forall x \in \mathbb{R}^{n},
$$

then

$$
x^{T} y=x^{T} P^{T} P y=(P x)^{T} P y=0 \quad \forall y \in \operatorname{Im}(A),
$$

i.e., $x \in \operatorname{Im}^{\perp}(A)$.

Summarizing the above, we conclude that

$$
x \in \operatorname{Im}^{\perp}(A) \Leftrightarrow P x=\left[0, \ldots, 0, x_{s+1}, \ldots, x_{n}\right]^{T} .
$$

Since

$$
P\left[I_{n}-A^{+} A\right] P^{T}=\left[\begin{array}{ll}
0 & \\
& I_{n-s}
\end{array}\right],
$$

a straightforward computation shows

$$
P\left[I_{n}-A^{+} A\right] P^{T}(P x)=P\left[I_{n}-A^{+} A\right] x=P x .
$$

Hence $\left[I_{n}-A^{+} A\right] x=x$. 
Lemma 5.6. Assume that $\mathrm{A} 1$ and $\mathrm{A} 2$ hold. Then

$$
\inf _{u_{P} \in B(L / 2) \cap \operatorname{Im}^{\perp}(R(x))}\left[a_{2}(x)+b^{T}(x) u_{P}\right]=\inf _{v \in B(L / 2)}\left[a_{2}(x)+\bar{b}^{T}(x) v\right],
$$

where $\bar{b}(x)=\left(I-R^{+}(x) R(x)\right) b(x)$.

Proof. From item 2 of Lemma 5.5, for any $u_{P} \in \operatorname{Im}^{\perp}(R(x))$,

$$
u_{P}=\left(I-R^{+}(x) R(x)\right) u_{P} .
$$

Note that

$$
\left(I-R^{+}(x) R(x)\right)^{T}=\left(I-R^{+}(x) R(x)\right) .
$$

Then

$$
\begin{aligned}
\inf _{u_{P} \in B(L / 2) \cap \operatorname{Im}^{\perp}(R(x))}\left[a_{2}(x)+b^{T}(x) u_{P}\right] & \\
=\operatorname{unf}_{u_{P} \in B(L / 2) \cap \operatorname{Im}^{\perp}(R(x))}\left[a_{2}(x)\right. & \left.+\bar{b}^{T}(x) u_{P}\right] \\
& \geq \inf _{v \in B(L / 2)}\left[a_{2}(x)+\bar{b}^{T}(x) v\right] .
\end{aligned}
$$

On the other hand, by item 1 of Lemma 5.5 , for any $v \in B(L / 2) \subset \mathbb{R}^{m}$, we have

$$
\left(I-R^{+}(x) R(x)\right) v \in \operatorname{Im}^{\perp}(R(x))
$$

Let

$$
u_{P}=\left(I-R^{+}(x) R(x)\right) v
$$

Note that

$$
\left\|I-R^{+}(x) R(x)\right\| \leq 1
$$

for all $x \in \mathbb{R}^{n}$; then $v \in B(L / 2)$ implies $u_{P} \in B(L / 2)$. Therefore,

$$
\begin{aligned}
\inf _{v \in B(L / 2)}\left[a_{2}(x)+\right. & \left.\bar{b}^{T}(x) v\right] \\
= & \inf _{v \in B(L / 2)}\left[a_{2}(x)+b^{T}(x)\left(I-R^{+}(x) R(x)\right) v\right] \\
& \geq \inf _{u_{P} \in B(L / 2) \cap \operatorname{Im}^{\perp}(R(x))}\left[a_{2}(x)+b^{T}(x) u_{P}\right] .
\end{aligned}
$$

Summarizing the above arguments, we obtain the conclusion.

In terms of Lemma 5.6, inequality (4.12) is equivalent to

$$
\inf _{v \in B(L / 2)}\left[a_{2}(x)+\bar{b}^{T}(x) v\right]<0 \quad \forall x \neq 0 .
$$


From now on, we use inequality (5.12) instead of (4.12). Denote

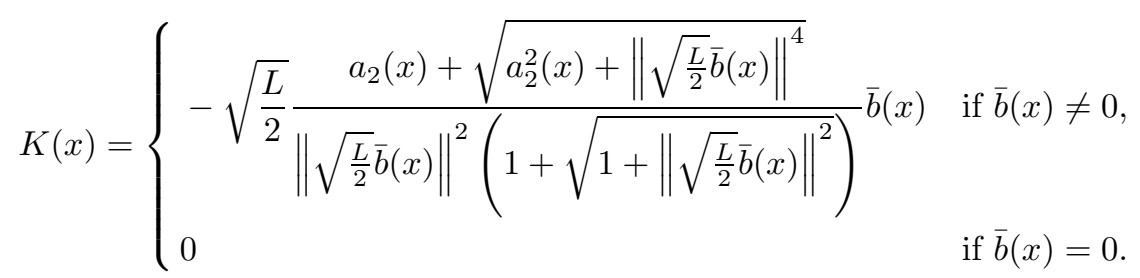

Then Proposition 2.3 yields the following result.

Theorem 5.7. If $\mathrm{A} 1, \mathrm{~A} 2$, and $\mathrm{A} 4$ hold, then a feasible control $u_{P}=$ $u_{P}(x)$ such that $u_{P}(0)=0$ is given by

$$
u_{P}(x)=\left(I-R^{+}(x) R(x)\right) K(x),
$$

which takes values in $B(L / 2)$ and is continuous in $\mathbb{R}^{n} \backslash\{0\}$. Moreover, if the LCF $V$ satisfies the small control property, then $u_{P}(x)$ is continuous in $\mathbb{R}^{n}$.

\section{Some illustrative EXamples}

In this section, we give some examples. The first two examples demonstrate the bounded continuous stabilizing control design technique obtained in the previous sections. The latter example shows that assumption A4 is only a sufficient condition of the existence of the continuous stabilizer, and not a necessary condition.

Example 6.1. Consider the system

$$
\left\{\begin{array}{l}
\dot{x}_{1}=\frac{x_{1}^{3} x_{2}^{2}}{\left(1+x_{1}^{2}+x_{2}^{2}\right)^{2}}+\frac{2 x_{1}^{3}+x_{1} x_{2}^{2}}{1+x_{1}^{2}+x_{2}^{2}} u_{1}+x_{2}^{2} u_{2}+x_{1} u_{1}^{2}+x_{1} u_{2}^{2}, \\
\dot{x}_{2}=-\frac{x_{2}^{5}}{\left(1+x_{1}^{2}+x_{2}^{2}\right)^{2}}+\frac{x_{1}^{2} x_{2}}{1+x_{1}^{2}+x_{2}^{2}} u_{1}+x_{1} x_{2} u_{2}+x_{2} u_{1}^{2}+x_{2} u_{2}^{2},
\end{array}\right.
$$

where $x=\left[\begin{array}{ll}x_{1} & x_{2}\end{array}\right]^{T} \in \mathbb{R}^{2}$ is the state and

$$
u=\left[\begin{array}{ll}
u_{1} & u_{2}
\end{array}\right]^{T} \in B(2)=\left\{u \in \mathbb{R}^{2} \mid\|u\|=u_{1}^{2}+u_{2}^{2}<2\right\}
$$

is the control input.

Take $V=\frac{1}{2}\left(x_{1}^{2}+x_{2}^{2}\right)$. Then

$$
\begin{aligned}
\left.\dot{V}\right|_{(6.1)}=\frac{x_{1}^{4} x_{2}^{2}-x_{2}^{6}}{\left(1+x_{1}^{2}+x_{2}^{2}\right)^{2}}+\frac{2\left(x_{1}^{4}+x_{1}^{2} x_{2}^{2}\right)}{1+} x_{1}^{2}+x_{2}^{2} & u_{1} \\
& +2 x_{1} x_{2}^{2} u_{2}+\left(x_{1}^{2}+x_{2}^{2}\right) u_{1}^{2}+\left(x_{1}^{2}+x_{2}^{2}\right) u_{2}^{2} .
\end{aligned}
$$


Using the notation of the previous sections, we have

$$
\begin{gathered}
a(x)=\frac{x_{1}^{4} x_{2}^{2}-x_{2}^{6}}{\left(1+x_{1}^{2}+x_{2}^{2}\right)^{2}}, \quad b(x)=\left[\begin{array}{c}
\frac{2\left(x_{1}^{4}+x_{1}^{2} x_{2}^{2}\right)}{1+x_{1}^{2}+x_{2}^{2}} \\
2 x_{1} x_{2}^{2}
\end{array}\right], \\
R(x)=\left[\begin{array}{cc}
x_{1}^{2}+x_{2}^{2} & 0 \\
0 & x_{1}^{2}+x_{2}^{2}
\end{array}\right] .
\end{gathered}
$$

Obviously, $R(x)>0$ for all $x \neq 0$. We set

$$
u_{1}=-x_{1}^{2} /\left(1+x_{1}^{2}+x_{2}^{2}\right), \quad u_{2}=0 .
$$

Then

$$
\left.\dot{V}\right|_{(6.1)}=-\frac{x_{1}^{6}+x_{2}^{6}}{\left(1+x_{1}^{2}+x_{2}^{2}\right)^{2}}<0 \quad \forall x \neq 0 .
$$

Moreover, note that $\|u\|^{2}=u_{1}^{2}+u_{2}^{2}<1$ and, therefore, $V$ is a Lyapunov control function for system (6.1). It is easy to see that $u=\left[\begin{array}{ll}u_{1} & u_{2}\end{array}\right]^{T}$ is continuous in $\mathbb{R}^{2}$ and $u(0)=0$ and, therefore, the LCF $V$ satisfies the small control property. Therefore, according to Theorem 3.2 , system (6.1) is globally asymptotically stabilized by the state feedback control $u_{0}=u_{0}(x)$ with $u_{0}(0)=0$, where

$$
\begin{aligned}
u_{0}(x)=-\frac{1}{2} \frac{\sqrt{2\left(x_{1}^{2}+x_{2}^{2}\right)}}{\sqrt{2\left(x_{1}^{2}+x_{2}^{2}\right)}+\sqrt{\frac{x_{1}^{4}}{1+x_{1}^{2}+x_{2}^{2}}+\frac{x_{1}^{2} x_{2}^{4}}{x_{1}^{2}+x_{2}^{2}}-\frac{x_{1}^{4} x_{2}^{2}-x_{2}^{6}}{\left(1+x_{1}^{2}+x_{2}^{2}\right)^{2}}}} \\
\cdot\left[\begin{array}{c}
\frac{2 x_{1}^{2}}{1+x_{1}^{2}+x_{2}^{2}} \\
\frac{2 x_{1} x_{2}^{2}}{x_{1}^{2}+x_{2}^{2}}
\end{array}\right] \quad \forall x \neq 0,
\end{aligned}
$$

which takes values in $B(L)$ and is continuous in $\mathbb{R}^{2}$.

Example 6.2. Consider the following system similar to system (6.1):

$$
\left\{\begin{array}{l}
\dot{x}_{1}=\frac{x_{1}^{3} x_{2}^{2}}{\left(1+x_{1}^{2}+x_{2}^{2}\right)^{2}}+\frac{2 x_{1}^{3}+x_{1} x_{2}^{2}}{1+x_{1}^{2}+x_{2}^{2}} u_{1}+x_{2}^{2} u_{2}+x_{1} u_{1}^{2}, \\
\dot{x}_{2}=-\frac{x_{2}^{5}}{\left(1+x_{1}^{2}+x_{2}^{2}\right)^{2}}+\frac{x_{1}^{2} x_{2}}{1+x_{1}^{2}+x_{2}^{2}} u_{1}+x_{1} x_{2} u_{2}+x_{2} u_{1}^{2},
\end{array}\right.
$$

where $x=\left[\begin{array}{ll}x_{1} & x_{2}\end{array}\right]^{T} \in \mathbb{R}^{2}$ is the state and

$$
u=\left[\begin{array}{ll}
u_{1} & u_{2}
\end{array}\right]^{T} \in B(2)=\left\{u \in \mathbb{R}^{2} \mid\|u\|=u_{1}^{2}+u_{2}^{2}<2\right\}
$$

is the control input.

We take $V=\frac{1}{2}\left(x_{1}^{2}+x_{2}^{2}\right)$. Then

$$
\left.\dot{V}\right|_{(6.2)}=\frac{x_{1}^{4} x_{2}^{2}-x_{2}^{6}}{\left(1+x_{1}^{2}+x_{2}^{2}\right)^{2}}+\frac{2\left(x_{1}^{4}+x_{1}^{2} x_{2}^{2}\right)}{1+x_{1}^{2}+x_{2}^{2}} u_{1}+2 x_{1} x_{2}^{2} u_{2}+\left(x_{1}^{2}+x_{2}^{2}\right) u_{1}^{2} \text {. }
$$


Therefore,

$$
\begin{gathered}
a(x)=\frac{x_{1}^{4} x_{2}^{2}-x_{2}^{6}}{\left(1+x_{1}^{2}+x_{2}^{2}\right)^{2}}, \quad b(x)=\left[\begin{array}{c}
\frac{2\left(x_{1}^{4}+x_{1}^{2} x_{2}^{2}\right)}{1+x_{1}^{2}+x_{2}^{2}} \\
2 x_{1} x_{2}^{2}
\end{array}\right], \\
R(x)=\left[\begin{array}{cc}
x_{1}^{2}+x_{2}^{2} & 0 \\
0 & 0
\end{array}\right] .
\end{gathered}
$$

Obviously, $R(x) \geq 0$, i.e., assumption A2 holds. As in Example 6.1, we can prove that $V$ satisfies inequality (4.1), i.e., assumption A1 and the small control property hold. Clearly,

$$
R^{+}(x)=\left[\begin{array}{cc}
\frac{1}{x_{1}^{2}+x_{2}^{2}} & 0 \\
0 & 0
\end{array}\right], \quad\left(R^{1 / 2}\right)^{+}(x)=\left[\begin{array}{cc}
\frac{1}{\sqrt{x_{1}^{2}+x_{2}^{2}}} & 0 \\
0 & 0
\end{array}\right] \quad \forall x \neq 0 .
$$

Hence $\left(R^{1 / 2}\right)^{+}(x)$ is continuous in $\mathbb{R}^{2} \backslash\{0\}$, i.e., assumption A4 holds.

A straightforward computation shows that

$$
\frac{1}{2}\left\|\left(R^{1 / 2}\right)^{+}(x) b(x)\right\|=\frac{x_{1}^{2} \sqrt{x_{1}^{2}+x_{2}^{2}}}{1+x_{1}^{2}+x_{2}^{2}} .
$$

Note that

and, therefore,

$$
\left\|R^{1 / 2}(x)\right\|=\sqrt{x_{1}^{2}+x_{2}^{2}}
$$

Then we have

$$
\frac{1}{2}\left\|\left(R^{1 / 2}\right)^{+}(x) b(x)\right\| \leq\left\|R^{1 / 2}(x)\right\|
$$

$$
\eta(x)=a(x)-\frac{1}{4} b^{T}(x) R^{+}(x) b(x)=-\frac{x_{1}^{6}+x_{2}^{6}}{\left(1+x_{1}^{2}+x_{2}^{2}\right)^{2}} .
$$

It is easy to see that

$$
b_{P}(x)=\left[\begin{array}{ll}
0 & 2 x_{1} x_{2}^{2}
\end{array}\right]^{T}, \quad a(x)-\left\|b_{P}(x)\right\|=\frac{x_{1}^{4} x_{2}^{2}-x_{2}^{6}}{\left(1+x_{1}^{2}+x_{2}^{2}\right)^{2}}-2\left|x_{1}\right| x_{2}^{2} .
$$

If $x_{1} \neq 0$, then

$$
\tau=\tau(x)=\frac{2 x_{1}^{2}\left(x_{1}^{2}+x_{2}^{2}\right)}{1+x_{1}^{2}+x_{2}^{2}}, \quad \beta=\beta(x)=x_{1}^{2}+x_{2}^{2}, \quad \gamma=\gamma(x)=2\left|x_{1}\right| x_{2}^{2} .
$$

Hence

$$
\xi(x)=\min \left\{-\frac{x_{1}^{6}+x_{2}^{6}}{\left(1+x_{1}^{2}+x_{2}^{2}\right)^{2}}, \frac{x_{1}^{4} x_{2}^{2}-x_{2}^{6}}{\left(1+x_{1}^{2}+x_{2}^{2}\right)^{2}}-2\left|x_{1}\right| x_{2}^{2}, \tilde{F}\left(x, z_{0}\right)\right\},
$$

where $z_{0}$ is a unique real solution of Eq. (5.5) satisfying $z_{0} \in(0,1)$, where $\tau, \beta$, and $\gamma$ are from (6.3). In turn,

$$
a_{1}(x)=\frac{x_{1}^{4}\left(x_{1}^{2}+x_{2}^{2}\right)}{\left(1+x_{1}^{2}+x_{2}^{2}\right)^{2}}-\mu(x) \xi_{\alpha}(x),
$$




$$
a_{2}(x)=-\frac{x_{1}^{6}+x_{2}^{6}}{\left(1+x_{1}^{2}+x_{2}^{2}\right)^{2}}-\mu(x) \xi_{\alpha}(x) .
$$

According to Theorems 5.4 and 5.7, we have $u_{I}=u_{I}(x)$ and $u_{P}=u_{P}(x)$, respectively, as follows:

$$
u_{I}(x)= \begin{cases}-\frac{1}{2} \frac{\sqrt{x_{1}^{2}+x_{2}^{2}}}{\sqrt{x_{1}^{2}+x_{2}^{2}}+\sqrt{\mu(x) \xi_{\alpha}(x)}}\left[\frac{2 x_{1}^{2}}{1+x_{1}^{2}+x_{2}^{2}}\right. & 0]^{T} \\ 0 & \text { if } x \neq 0, \\ \text { if } x=0\end{cases}
$$

and

$$
u_{P}(x)= \begin{cases}-\frac{a_{2}(x)+\sqrt{a_{2}^{2}(x)+16 x_{1}^{4} x_{2}^{8}}}{4 x_{1}^{2} x_{2}^{4}\left(1+\sqrt{1+4 x_{1}^{2} x_{2}^{4}}\right)}\left[\begin{array}{ll}
0 & 2 x_{1} x_{2}^{2}
\end{array}\right]^{T} & \text { if } x_{1} x_{2} \neq 0 \\
0 & \text { if } x_{1} x_{2}=0 .\end{cases}
$$

Therefore, system (6.2) is globally asymptotically stabilized by the bounded continuous state feedback control $u=u_{I}(x)+u_{P}(x)$, where $u_{I}(x)$ and $u_{P}(x)$ are as above.

Example 6.3. Consider the following system:

$$
\left\{\begin{array}{l}
\dot{x}_{1}=\frac{x_{1} x_{2}^{4}}{\left(1+x_{1}^{2}\right)^{2}}+\frac{2 x_{1}^{3}}{1+x_{1}^{2}} u_{1}+x_{1} u_{1}^{2}+x_{2} u_{2}^{2}, \\
\dot{x}_{2}=-\frac{x_{2}^{5}}{\left(1+x_{1}^{2}\right)^{2}}+\frac{x_{2}^{3}}{1+x_{1}^{2}} u_{1}+\left(x_{2}-x_{1}\right) u_{2}^{2},
\end{array}\right.
$$

where $x=\left[\begin{array}{ll}x_{1} & x_{2}\end{array}\right]^{T} \in \mathbb{R}^{2}$ is the state and

$$
u=\left[u_{1} u_{2}\right]^{T} \in B(1)=\left\{u \in \mathbb{R}^{2} \mid\|u\|=u_{1}^{2}+u_{2}^{2}<1\right\}
$$

is the control input.

By the state feedback control law,

$$
u_{1}=-\frac{x_{1}^{2}}{1+x_{1}^{2}}, \quad u_{2}=0
$$

the closed-loop system of system (6.4) is

$$
\dot{x}_{1}=-\frac{x_{1} x_{2}^{4}-x_{1}^{5}}{\left(1+x_{1}^{2}\right)^{2}}, \quad \dot{x_{2}}=-\frac{x_{1}^{2} x_{2}^{3}+x_{2}^{5}}{\left(1+x_{1}^{2}\right)^{2}} .
$$

We take $V=\frac{1}{2}\left(x_{1}^{2}+x_{2}^{2}\right)$. Then a straightforward computation shows that

$$
\left.\dot{V}\right|_{(6.6)}=-\frac{x_{1}^{6}+x_{2}^{6}}{1+x_{1}^{2}}<0 \quad \forall x \neq 0
$$

Hence (6.5) is a bounded continuous stabilizer of system (6.4) and $V=$ $\frac{1}{2}\left(x_{1}^{2}+x_{2}^{2}\right)$ is a Lyapunov control function of system (6.4) satisfying the small control property. In the previous notation, it is easy to see that $R(x)=$ $\operatorname{diag}\left(x_{1}^{2}, x_{2}^{2}\right) \geq 0$ for all $x \in \mathbb{R}^{2}$. Therefore, $\left(R^{1 / 2}\right)^{+}(x)$ is not continuous in $\mathbb{R}^{2} \backslash\{0\}$, i.e., assumption A4 does not hold. Therefore, assumption A4 is 
only a sufficient condition of the existence of the continuous stabilizer, and not a necessary condition.

\section{Conclusion}

This paper considered the stabilization of quadratic-input nonlinear systems with bounded controls. According to the type of quadratic-input forms, both positive definite and positive semi-definite cases are considered. For the case of positive definiteness, we gave a universal formula for the bounded continuous stabilizers of quadratic-input nonlinear systems with bounded controls via a known Lyapunov control function. For the case of positive semi-definiteness, a constructive parametrization of bounded continuous stabilizers was presented under the assumption that there exists a known Lyapunov control function with respect to the set $B(L / 2)$ rather than $B(L)$. This is our basic assumption. In our approach, the factor $1 / 2$ is maximum in $B(L / 2)$ in inequality (4.1) and the definition of $\xi(x)$, and cannot be relaxed to, say, $\rho<1$. In fact, if $B(L / 2)$ is replaced by $B(\rho L)$ with $\rho<1$, then $u_{I} \in B(\rho L) \cap \operatorname{Im}(R(x))$ and $u_{P} \in B(\rho L) \cap \operatorname{Im}^{\perp}(R(x))$. In turn, $u=u_{I}+u_{P} \in B(2 \rho L)$. To assure that this $u$ is in the admissible control set, $B(L), \rho$ should satisfy $\rho \leq 1 / 2$. Moreover, some illustrative examples were included.

\section{REFERENCES}

1. Z. Artstein, Stabilization with relaxed control. Nonlinear Anal., Theory, Meth., Appl. 7 (1983), No. 11, 1163-1173.

2. J. W. Curtis and R. W. Beard, Satisficing: a new approach to constructive nonlinear control. IEEE Trans. Automat. Control 49 (2004), No. 7, 1090-1102.

3. W. Lin, Bounded smooth state feedback and global separation principle for nonaffine nonlinear systems. Syst. Control Lett. 26 (1995), No. 1, $41-53$.

4. $ـ$ Global asymptotic stabilization of general nonlinear systems with stable free dynamics via passivity and bounded feedback. Automatica 32 (1996), No. 6, 915-924.

5. Y. Lin and E. D. Sontag, A universal formula for stabilization with bounded control. Syst. Control Lett. 16 (1991), No. 6, 393-397.

6. M. Malisoff and E. D. Sontag, Universal formulas for LCF's with respect to Minkowski norms. Syst. Control Lett. 40 (2000), No. 4, 246-260.

7. E. Moulay and W. Perruquetti, Stabilization nonaffine systems: a constructive method for polynomial systems. IEEE Trans. Automat. Control 50 (2005), No. 4, 520-526.

8. R. Penrose, A generalized inverse for matrices. Proc. Cambridge Phil. Soc. 51 (1955), 406-413. 
9. E. D. Sontag, A universal construction of Arstsein's theorem on nonlinear stabilization. Syst. Control Lett. 13 (1989), No. 2, 117-123.

10. R. Suárez, J. Solís-Duan, and B. Aguirre, Global LCF stabilization for systems with compact convex control value sets. In: Proc. 40th Conf. Decision Control, Orlando, Florida USA (2001), pp. 3838-3843.

11. R. Suárez, J. Solís-Duan, and J. Álvarez-Ramírez, Global robust stabilization of nonlinear systems subject to input constraints. Int. J. Robust Nonlinear Control 12 (2002), No. 14, 1227-1238.

12. J. Zhong, D. Cheng, and X. Hu, Constructive stabilization for quadratic input nonlinear systems. Submitted to Automatica.

(Received May 03 2007, received in revised form June 07 2007)

Authors' addresses:

Jianghua Zhong

Institute of Systems Science, Chinese Academy of Sciences, Beijing 100080, P.R.China

Daizhan Cheng

Institute of Systems Science, Chinese Academy of Sciences, Beijing 100080, P.R.China

E-mail: dcheng@iss.ac.cn

Xiaoming $\mathrm{Hu}$

Optimization and Systems Theory, Royal Institute of Technology, Stockholm, SWEDEN

E-mail: hu@math.kth.se 